\title{
Recognization of Cloth Pattern and Colour for Blind People
}

\author{
G. Siva Sankar Varma ${ }^{1}$, Shirisha Kamishetti ${ }^{2}$, P. Sudha ${ }^{3}$,H. Lavanya ${ }^{4}$, E. Sowmya Vani ${ }^{5}$ \\ ${ }^{1}$ Assistant professor-Electronics and communication Engineering, BVRIT Hyderabad College of Engineering for Women \\ Bachupally, Hyderabad, Telangana, India \\ 2,3,4,5 UG student -Electronics and communication Engineering, BVRIT Hyderabad College of Engineering for Women \\ Bachupally, Hyderabad, Telangana, India
}

\begin{abstract}
As we know that recognizing cloth pattern and colour of a cloth is easy for a person but, it is a difficult task for visually impaired people i.e blind people. This Recognization system will help the visually impaired people to recognize the pattern and colour of the cloth. But this is also a challenging research problem due to rotation, scaling, illumination, and especially large intra-class pattern variations. We have developed a system that recognizes clothing patterns in four main categories (plaid, striped, pattern-less, and irregular) and identifies 8 clothing colors. By using algorithms like SIFT and Classifier like SVM and Discrete Wavelet Transform, Radon Transform to detect the color and Pattern respectively. To evaluate the effectiveness of the proposed approach, we used the CCNY Clothing Pattern dataset.
\end{abstract}

Keywords - Visually impaired People, recognizing cloth pattern and colour, SIFT algorithm, SVM Classifier, Discrete Wavelet Transform, Radon Transform.

\section{INTRODUCTION}

Visual impairment leads to loss of livelihood and difficulty not only to themselves but to the families they support. Most blind children don't have access to extra special teaching aids they need to learn. Based on data from the World Health Organization (WHO), there are more than 37 million people across the globe who are blind, over 15 million are in India [1]. And according to WHO in 2012 about 2.2 Billion people out of whole population are visually impaired in which almost $50 \%$ can be solved with proper medical care. For visually impaired people when they go to the textiles for selecting the dresses, they could not be able to choose the clothes. So, choosing clothes with suitable colors and patterns is very difficult for them. They can manage this difficulty with the help of other people. Some of them use plastic Braille labels or different types of electronic assistance but they cost high. Most of the blind people due to these difficulties oh high cost they prefer to wear the clothes with a uniform color or without any pattern. The visually impaired people have difficulty for choosing the clothes.

Our System helps the blind i.e. visually impaired people to detect the pattern of the cloth and color of the cloth using the SIFT Algorithm technique which an object is recognized in a new image by individually comparing each feature from the new image to this database based on the Euclidean distance between their feature vectors [2].The SVM Classifier which classifies it into one among the given four patterns as the output and if the given image is not matching any of the pattern in the trained dataset it will not give the pattern type it will just give the colour output.

\section{WORKING PROCESS}

The Input Image Is taken/selected from the dataset and the pre-processing of the image is done using sift algorithm then the features of the image are determined and will be go through the SVM classifier which will classify on the basis of the pre-processed training set and will detect the colour of the image cloth input through audio and then it will give us the DWT transform which helps us finding the pattern of the cloth and will give the output displayed on the screen and also output in audio output.

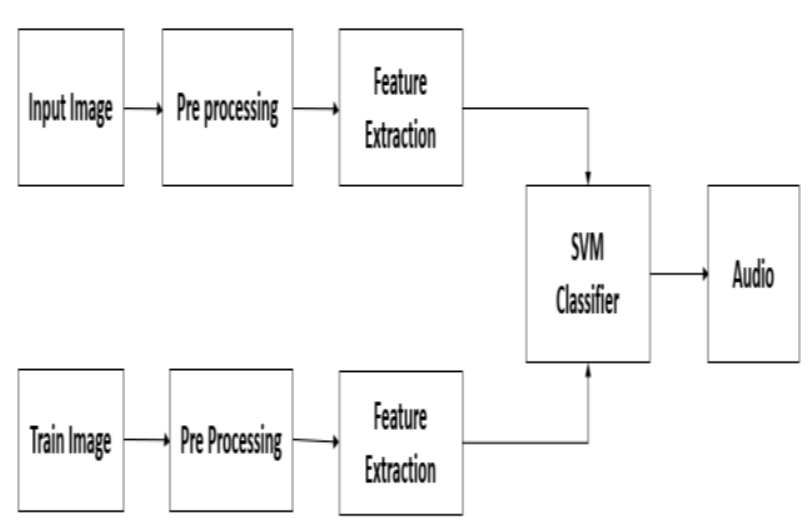

Fig. 1. Block Diagram of the Process

\section{SIFT ALGORITHM}

In, SIFT (Scale Invarient Feature Transform) algorithm key points of objects are extracted from a given image and compared with stored images in the database. An object is recognized in a new image by individually comparing each feature from the new image to this database based on the Euclidean distance between their feature vectors. Image content is transformed into local feature coordinates that are invariant to translation, rotation, scale and other image properties [3]. These parameters are compared with all images in data set and finds for exact match. 


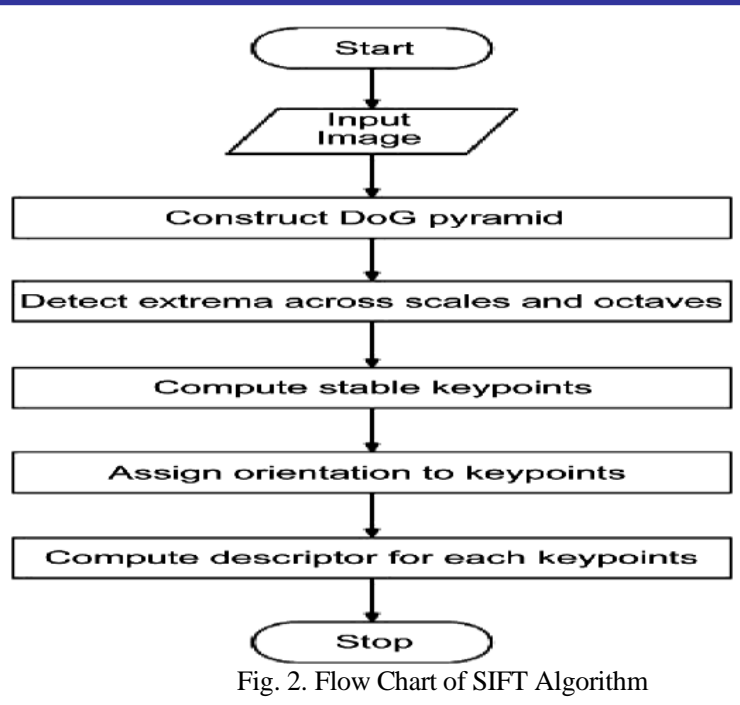

\section{A. SVM Classifier}

Several recent studies have reported that the SVM (support vector machines) generally are capable of delivering higher performance in terms of classification accuracy than the other data classification algorithms [3]. A wide range of real-world problems such as text categorization, hand-written digit recognition, tone recognition, image classification and object detection, micro-array gene expression data analysis, data classification. However, for some datasets, the performance of SVM is very sensitive to how the cost parameter and kernel parameters are set. As a result, the user normally needs to conduct extensive cross validation in order to figure out the optimal parameter setting. This process is commonly referred to as model selection. One practical issue with model selection is that this process is very time consuming [4]. We have experimented with a number of parameters associated with the use of the SVM algorithm that can impact the results. These parameters include choice of kernel functions, the standard deviation of the Gaussian kernel, relative weights associated with slack variables to account for the non-uniform distribution of labeled data, and the number of training examples.

Let us consider the four different Patterns data set such as Plaid, Irregular, Striped, Pattern less cloth type data which all have different features, classes, number of training data and different number of testing data. These all data taken from CCNY data set.

\section{B. Wavelet Feature Extraction}

Wavelet transform is a local transformation of space and frequency which can effectively extract information from the images for texture analysis. The wavelet series expansion of function can be defined with respect to a scaling function and a wavelet function with different scaling and wavelet coefficients [5]. With the coefficients we can easily obtain the different levels of wavelet decomposition. In our system, we use DWT (Discrete Wavelet Transform).

DWT: The Discrete Wavelet Transform (DWT), which is based on sub-band coding, is found to yield a fast computation of Wavelet Transform. It is easy to implement and reduces the computation time and resources required. In many wavelets transform methods the signals are analyzed using a set of basic functions which relate to each other by simple scaling and translation. In the case of DWT, a timescale representation of the digital signal is obtained using digital filtering techniques. The signal to be analyzed is passed through filters with different cut-off frequencies at different scales.

\section{RESULT}

The results of this system will show the DWT (Discrete Wavelet Transform) of the selected input image and then it will speak out the colour of the given cloth if it is single colour or mixture of colours and the colours involved in it. Later, it will show us the Radon Transform the given input image and will display the output as the pattern as well as voice output of the cloth pattern.

Below are the Experimental outputs of the system for different pattern inputs:

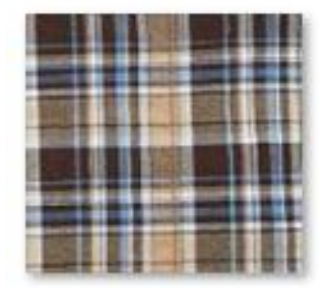

Fig. 3. Plaid Type (Input-1)

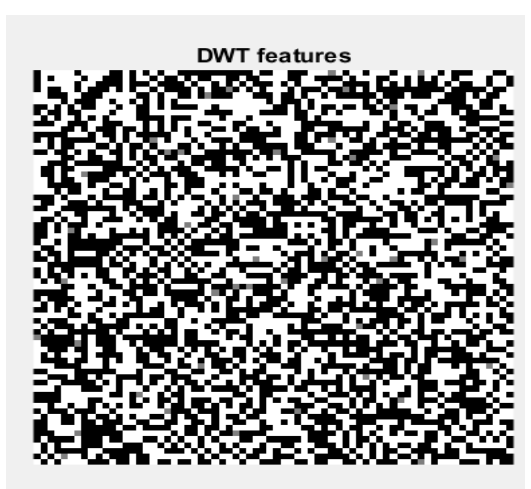

Fig. 4. DWT of Plaid Cloth Type

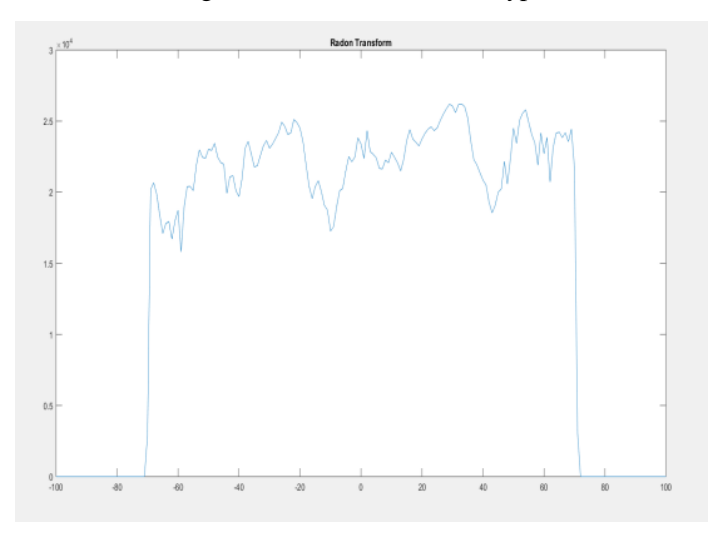

Fig. 5. Randon Transform of Plaid Cloth Type 


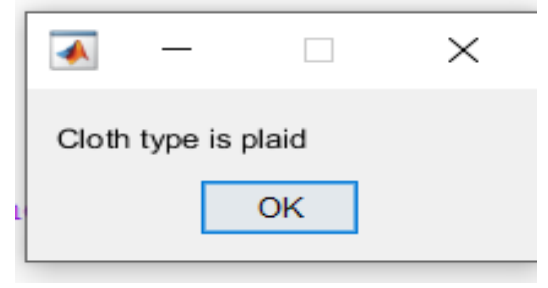

Fig. 6. Plaid Type Output

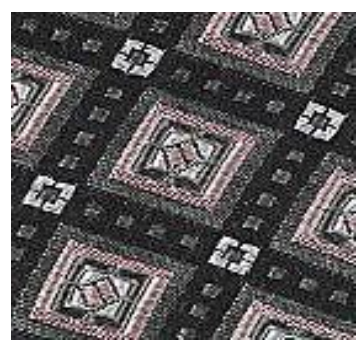

Fig. 7. Irregular Type (Input-2)

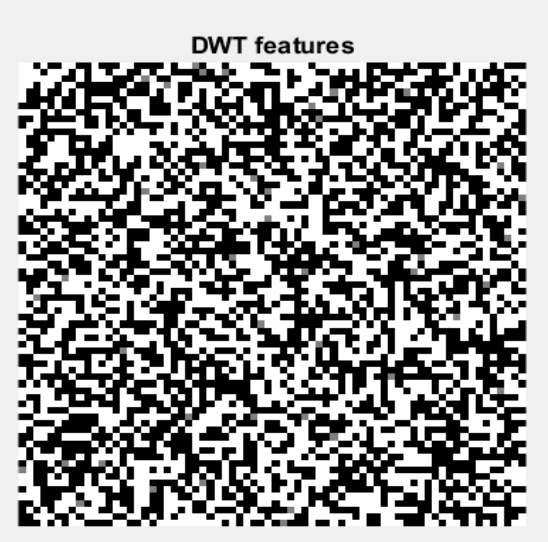

Fig. 8. DWT of Irregular Cloth Type

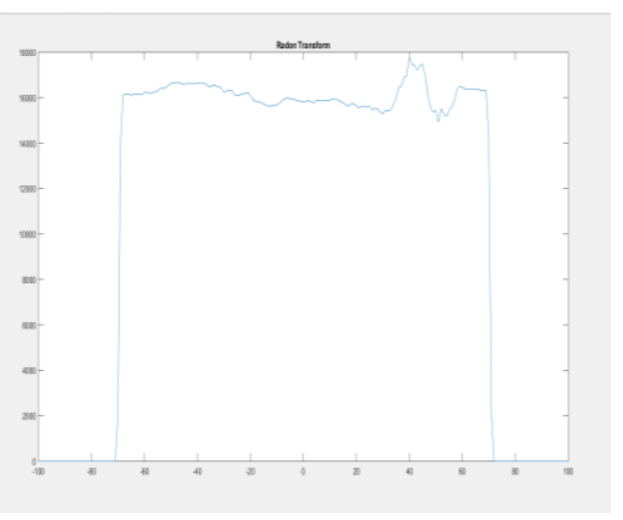

Fig. 9. Randon Transform of Irregular Cloth Type

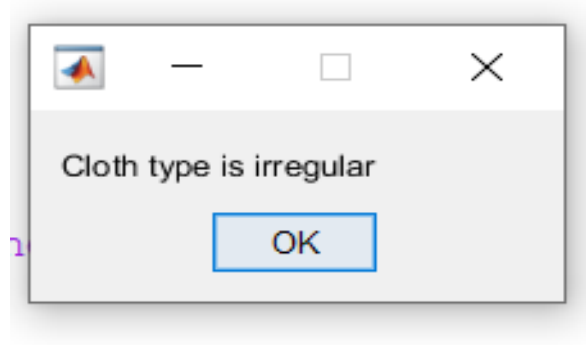

Fig. 10. Irregular pattern Output

\section{CONCLUSION}

This paper proposes a system "Recognization of Cloth Pattern and Colour for blind people" which can be used to recognize the pattern and colour of the cloth. The system method provides a simple and reliable method for recognizing the pattern and the colour. The images are taken from CCNY database in order to implement the proposed system. All data were pre-processed and the feature of the images can be distinguished. This development of automatic recognizing clothing pattern system capable of real time identification of the pattern and the color in the cloth.

\section{FUTURE SCOPE}

This System is more efficient and accurate to recognize the patterns because it can easily classify with large number of datasets, number of operations by reducing number of samples to be processed which results in reduced processing time required to be processed. We are also working to add more colours and patterns to the system.

\section{REFERENCES}

[1] Kocur, R. Parajasegaram, and G. Pokharel, "Global data on visual impairment in the year 2002," Bulletin World Health Org., 2004.

[2] G. Siva Sankar Varma, D. Venkata Siva Prasad, R. Akhila, V. Sai Sahana Sri, "Product Label Reading Using Sift Algorithm to Assist Visually Impaired", International Journal of Management, Technology and Engineering, Vol 8, Issue XII, ISSN: 2249-7455, pp 4095-4097, December 2018.

[3] Clothing Color and Pattern Recognition for Visually Impaired People, Shruti Bharadwaj,Sharath H.K., Praveena M.B., Ajay Shetty,Shivarudraiah B, International Journal ofEngineering, Management \&Sciences(IJEMS) ISSN-2348-3733, Volume-2, pp 104-107, Issue-5,May 2015.

[4] Data lassification: A Rough - SVM Approach, D. K. Srivastava, K S. Patnaik, L. Bhambhu, Contempororary Engineering Sciences, Vol 3, no.2, pp 77-86, January 2010.

[5] Colour and Pattern Recognition for Visually Impaired People with Kinect Sensor K.Likitha Raj, J. Amarendra, M.Tech(Ph.d), Department of Electronics and communication Engineering, Audisankara College of Engg \& Tech (Autonomous), International Journal for Research in Applied Science \& Engineering Technology, (IJRASET), Volume 3, Special Issue-1, IC-Value:13.98, ISSN: 23219653, pp 63-68, May 2015. 